\title{
Fast implementation for modified adaptive multi-pulse compression
}

\author{
Xianxiang Yu ${ }^{1}$, Guolong Cui ${ }^{1 *}$, Meifang Luo ${ }^{2}$, Lingjiang Kong ${ }^{1}$ and Dan Ran $^{3}$
}

\begin{abstract}
This paper deals with the estimation of range-Doppler plane in pulse Doppler radar system, accounting both for clutter-free scenario and clutter scenario. A modified adaptive multi-pulse compression (MAMPC) algorithm including the estimation stages of range dimension and Doppler dimension is proposed for clutter-free scenario, where each stage is implemented based on the gain constraint adaptive pulse compression (GCAPC) algorithm. Additionally, the combination of whitening method removing the correlation of clutter component and MAMPC algorithm is presented for the considered clutter scenario. Numerical simulations are provided to validate the effectiveness of MAMPC in terms of estimation of range-Doppler plane and computation burden.
\end{abstract}

Keywords: Range-Doppler plane, Modified adaptive multi-pulse compression, Range dimension, Doppler dimension, Gain constraint adaptive pulse compression

\section{Introduction}

Traditionally, the pulse Doppler radar systems repeat the same waveform to allow efficient pulse compression and Doppler processing technique to be used [1]. The traditional pulse compression method is matched filtering, in which the high range sidelobe of strong targets may interfere or even mask nearby weak targets. The Doppler processing technique, such as the moving target detection (MTD), also obtains Doppler sidelobe that results in the masking problem [2]. Consequently, suppressing the range-Doppler sidelobe is meaningful for target detection.

Suppressing range or Doppler sidelobe has been received considerable attention. Summarizing, these works can be classified into three categories. The first category deals with the problem of adaptive range sidelobe suppression. In [3], iterative reweighted least squares (IRLS) algorithm was used to suppress range sidelobe. In [4], several binary pulse compression codes were designed to greatly reduce sidelobe meanwhile suffering only a small $\mathrm{S} / \mathrm{N}$ loss. In [5], the adaptive pulse compression (APC) was proposed, which was shown to successfully suppress the range sidelobes over a variety of

*Correspondence: cuiguolong@uestc.edu.cn

${ }^{1}$ University of Electronic Science and Technology of China, 2006 Xi Yuan Ave, West Hi-tech Zone, Chengdu, China

Full list of author information is available at the end of the article stressing scenarios. Li et al. [6] has demonstrated that gain-constraint-APC (GCAPC) [7] has better estimating performance especially for weak targets compared to original APC algorithm [5].

The second category focuses on addressing the problem of Doppler sidelobe suppression. As the mathematical model of the Doppler estimation for coherent multipulses is similar to direction of arrival (DOA) estimation, the studies related to DOA estimation can also be used in Doppler sidelobe suppression. For instance, the most well-known methods for DOA estimation are MUSIC [8], root-MUSIC [9] and ESPRIT [10]. Re-iterative super resolution (RISR) was studied in [11, 12], which was used to estimate DOA in array signal processing firstly.

The third category studies the sidelobe suppression problem by jointly suppressing range-Doppler sidelobe [13, 14]. In [15], two-dimensional reiterative minimum mean square error (MMSE) and 2-D least square (LS) solutions that mitigate the sidelobe of both pulse compression processing and antenna radiation patterns are derived. In [16], a RISR algorithm was used in conjunction with Golay waveforms for range-Doppler estimation. In [17], a recursive MMSE-based time-range adaptive processing was proposed for the purpose of jointly suppressing the range-Doppler sidelobe. However, clutter 
scenario was not considered. In [18], the adaptive multipulse compression (AMPC) was presented to successfully suppress the range-Doppler sidelobe over a variety of stressing scenarios. Unfortunately, the high computational cost of this method limits its usage in real-time systems. It is worth noting that these approaches based dimensionality reduction are well known in open literature as a means to facilitate practical solutions to computation problems. In [19], the fast adaptive pulse compression (FAPC) was proposed. In [20], the fast adaptive multi-pulse compression (FAMPC) was proposed based on fast adaptive pulse compression (FAPC) by segmenting the MMSE cost function into blocks. Of course, some inherent loss in performance can generally be expected by reducing dimensionality, though the attendant reduction in computation often easily justifies the trade-off.

In this paper, we propose a modified adaptive multipulse compression (MAMPC) algorithm to obtain both good estimation performance and small amount of calculations. Unlike [21], we also consider clutter scenario assuming that some knowledge of clutter statistics is available. For clutter-free scenario, we implement MAMPC with two estimating stages by utilizing GCAPC algorithm. Specially, we obtain estimation in the range dimension using GCAPC. Then, based on the obtained results, we achieve the estimation of range-Doppler plan in the Doppler dimension by exploiting GCAPC. In particular, for clutter scenario, the combination of whitening method removing the correlation of clutter component and MAMPC algorithm is proposed. Simulation results highlight that MAMPC is capable of achieving a close estimation performance with that of AMPC, while shares much less computational time than AMPC.

The rest of the paper is organized as follows. In Section 2, we give the signal model of range-Doppler dimension. In Section 3 and Section 4, we present MAMPC algorithm for clutter free scenario and the combination of whitening method and MAMPC algorithm for clutter scenario, respectively. In Section 5, we evaluate the capabilities of MAMPC via numerical results. Finally, in Section 6, we provide some concluding remarks.

Notation: Vectors (matrices) are denoted by boldface lower (upper) case letters. Superscripts $(\cdot)^{T},(\cdot)^{*}$, and $(\cdot)^{H}$ denote transpose, complex conjugate, and complex conjugate transpose, respectively. $|\cdot|$ denotes the modulus of a complex number. $E(\cdot)$ is the statistical expectation. $\sum(\cdot)$ denotes the summation operation. $\mathbf{I}_{N}$ is the identity matrix with $N \times N$ demension. $\operatorname{diag}($.) is an operation that creates a diagonal matrix by using the input vector as its diagonal. Finally, $\otimes$ denotes the Kronecker product.

\section{Signal model}

Consider a stationary monostatic radar system which transmits $M$ coherent pulses of train. Let $s(t)$ be the baseband complex probing waveform. Assume that there are $Q$ point-like targets and $P$ clutter scatterers in different range cells and with different radial velocities. The received signal $y_{m}(t)$ of the $m$ th pulse can be represented as

$$
\begin{aligned}
& y_{m}(t)=\sum_{q=1}^{Q} \sigma_{T, m q} \exp \left(j 2 \pi(m-1) f_{q}\right) s\left(t-\tau_{T, q}\right) \\
& +\sum_{p=1}^{P} \sigma_{c, m p} s\left(t-\tau_{c, m p}\right)+b_{m}(t)
\end{aligned}
$$

where

- $\sigma_{T, m q}$ for $q=1, \cdots, Q$, denote the complex parameters accounting for the target radar cross section (RCS), channel propagation effects, and other terms involved into the radar range equation.

Assume that $\sigma_{T, m q}=\sigma_{T, q}$ for all $m=1,2, \cdots, M$, which are distributed as circular zero-mean complex Gaussian random variables. In other words, the pdf of the amplitude $A_{q}=\left|\sigma_{T, q}\right|$ is Rayleigh distributed, i.e.,

$$
p_{A_{q}}(x)=\frac{2 x}{\bar{\sigma}_{T, q}^{2}} \exp \left\{-\frac{x^{2}}{\bar{\sigma}_{T, q}^{2}}\right\}, x \geq 0,
$$

- $f_{q}=2 v_{q} T_{r} / \lambda$ denotes the normalized Doppler frequency of the $q$ th target while $v_{q}$ is the radial velocity and $\lambda$ is the carrier wavelength.

- $\tau_{T, q}$ and $\tau_{c, m q}$ denote, respectively, the two-way time delays for the $q$ th target and $p$ th clutter scatterer for the mth pulse.

- $\sigma_{c, m p}$ is the complex scattering parameter of the $p$ th clutter scatterer at the $m$ th pulse for $m=1, \cdots, M$ and $p=1, \cdots, P$.

- $b_{m}(t)$ denotes the zero-mean circular complex Gaussian random process.

Let $\mathbf{s}=\left[s_{1}, s_{2}, \ldots, s_{N}\right]^{T}$ be the discrete version of the baseband waveform $s(t)$, then, after sampling with the same rate, the discrete versions of the received signal $y_{m}(t)$ and the noise term $b_{m}(t)$ can be expressed respectively by

$$
\begin{aligned}
\mathbf{y}_{m} & =\left[y_{m}[1], y_{m}[2], \cdots, y_{m}[L]\right]^{T}, \\
\mathbf{b}_{m} & =\left[b_{m}[1], b_{m}[2], \cdots, b_{m}[L]\right]^{T},
\end{aligned}
$$

where $L$ denotes the number of the range cells. Stacking the $M$ pulses to be the columns of the $L \times M$-dimensional data matrix $\mathbf{Y}$, we have 


$$
\mathbf{Y}=\left[\mathbf{y}_{1}, \mathbf{y}_{2}, \cdots, \mathbf{y}_{M}\right] .
$$

In addition, let us divide uniformly the normalized frequency with $K$ points, e.g., $f_{k}=(k-1) / K$, $k=1, \cdots, K$, and denote by $\mathbf{f}=\left[f_{1}, f_{2}, \cdots, f_{K}\right]$ the $K$-points Doppler frequency vector. Hence, the $L \times K$ dimensional range-Doppler plane $\mathbf{X}$ accounting for the scattering coefficients of the targets of interest can be expressed as

$$
\mathbf{X}=\left[\begin{array}{cccc}
X[1,1] & X[1,2] & \cdots & X[1, K] \\
X[2,1] & X[2,2] & \cdots & X[2, K] \\
\vdots & \vdots & \ddots & \vdots \\
X[L, 1] & X[L, 2] & \cdots & X[L, K]
\end{array}\right]
$$

In this paper, we employ the adaptive pulse compression algorithm to estimate the components of the rangeDoppler plane $\mathbf{X}$. To this end, we formulate the sub-vector $\mathbf{y}_{m}[l]=\left[y_{m}[l], \cdots, y_{m}[l+N-1]\right]^{T}$ for the $m$ th pulse with length $N$ as

$$
\begin{aligned}
\mathbf{y}_{m}[l] & =\mathbf{G} \sum_{k=1}^{K} \overline{\mathbf{x}}_{l}[k] \exp \left(j 2 \pi(m-1) f_{k}\right)+\mathbf{G c}_{m}[l]+\mathbf{b}_{m}[l], \\
& =\mathbf{G X}[l] \mathbf{F}^{T}[m]+\mathbf{G c}_{m}[l]+\mathbf{b}_{m}[l]
\end{aligned}
$$

where

- $\mathbf{G}$ is the $N \times(2 N-1)$-dimensional linear transformation matrix, given by

$$
\mathbf{G}=\left[\begin{array}{ccccccc}
s_{N} & s_{N-1} & \cdots & s_{1} & & & 0 \\
& s_{N} & \cdots & s_{2} & s_{1} & & \\
& & \ddots & \vdots & \vdots & \ddots & \\
& & & s_{N} & s_{N-1} & \cdots & s_{1}
\end{array}\right] .
$$

- $\overline{\mathbf{x}}_{l}[k]=[X[l-N+1, k], \cdots, X[l+N-1, k]]^{T}$ is the $(2 N-1) \times 1$-dimensional sub-vector accounting the scattering coefficients at the $k$ th Doppler frequency in the range-Doppler plane and $\mathbf{X}[l]=\left[\overline{\mathbf{x}}_{l}[1], \cdots, \overline{\mathbf{x}}_{l}[K]\right]$ is the $(2 N-1) \times K$-dimensional sub-matrix of $\mathbf{X}$.

- $\mathbf{F}$ is the discrete Fourier transform matrix, given by

$$
\mathbf{F}=\left[\begin{array}{cccc}
1 & 1 & \cdots & 1 \\
1 & e^{j \frac{\pi \pi}{K} 1} & \cdots & e^{j \frac{2 \pi(K-1)}{K} 1} \\
\vdots & \vdots & & \vdots \\
1 & e^{j \frac{\pi}{K}(M-1)} & \cdots & e^{j \frac{2 \pi(K-1)}{K}(M-1)}
\end{array}\right]
$$

and $\mathbf{F}^{T}[m]$ denotes the $m$ th column of the matrix $\mathbf{F}^{T}$.

- $\mathbf{c}_{m}[l]=\left[c_{m}[l-N+1], \cdots, c_{m}[l+N-1]^{T}\right.$ is the $(2 N-1) \times 1$-dimensional sub-vector accounting the clutter scattering coefficients.
- $\mathbf{b}_{m}[l]=\left[b_{m}[l], \cdots, b_{m}[l+N-1]\right]^{T}$ is the $N \times 1$ dimensional sub-vector of $\mathbf{b}_{m}$, which is distributed as the complex circular zero-mean Gaussian random vector with identity covariance matrix $\sigma_{n}^{2} \mathbf{I}_{N}$.

Let us rewrite all the $M$ sub-vectors $\mathbf{y}_{m}[l]$, for $m=$ $1, \cdots, M$, in terms of the $N \times M$-dimensional matrix, we have

$$
\mathbf{Y}[l]=\mathbf{G X}[l] \mathbf{F}^{T}+\mathbf{G C}[l]+\mathbf{B}[l], l=1, \cdots, L,
$$

where $\mathbf{Y}[l]=\left[\mathbf{y}_{1}[l], \cdots, \mathbf{y}_{M}[l]\right], \mathbf{C}[l]=\left[\mathbf{c}_{1}[l], \cdots, \mathbf{c}_{M}[l]\right]$, and $\mathbf{B}[l]=\left[\mathbf{b}_{1}[l], \cdots, \mathbf{b}_{M}[l]\right]$.

\section{Fast implementation of MAMPC for clutter-free scenario}

In this section, we focus on the estimation of the components of range-Doppler plane $\mathbf{X}$ in the presence of white noise using the fast implementation of MAMPC. To this end, the signal model without considering clutter in Eq. (8) can be simplified as

$$
\mathbf{Y}[l]=\mathbf{G X}[l] \mathbf{F}^{T}+\mathbf{B}[l], l=1, \cdots, L .
$$

\subsection{The process in range dimension}

In this subsection, we are devoted to the process in range dimension by exploiting the proposed fast algorithm. Specifically, Eq. (8) can be further recast as

$$
\mathbf{Y}[l]=\mathbf{G A}[l]+\mathbf{B}[l],
$$

while

$$
\mathbf{A}[l]=\mathbf{X}[l] \mathbf{F}^{T},
$$

where $\mathbf{A}[l]=\left[\mathbf{a}_{1}[l], \cdots, \mathbf{a}_{M}[l]\right]$ is a $(2 N-1) \times M$ dimensional matrix and $\mathbf{a}_{m}[l]=\left[\alpha_{m}[l-(N-\right.$ $\left.1)], \ldots, \alpha_{m}[l+(N-1)]\right]^{T}$ denotes the $m$ th column of $\mathbf{A}[l]$, for $m=1, \ldots, M$. In particular, for the $m$ th pulse, we have

$$
\mathbf{y}_{m}[l]=\mathbf{G a}_{m}[l]+\mathbf{b}_{m}[l], l=N, \cdots, L-(N-1) .
$$

Since observation $\mathbf{y}_{m}[l]$ is the linear combination of $\mathbf{a}_{m}[l], \alpha_{m}[l]$ can be obtained by designing a filter for $l$ th range cell employing GCAPC algorithm. In the following, we focus on the discussion of three cases of different scopes of range cell for the estimation of $\alpha_{m}[l]$. Specifically, for $l=N, \cdots, L-(N-1)$, we minimize the output power of $l$ th range cell by devising GCAPC filter coefficients $\mathbf{w}_{m}[l]$ accounting for $\left\{\mathbf{w}_{m}[l]\right\}^{H} \mathbf{s}=1$, with corresponding to optimization problem formulated as

$$
\begin{aligned}
& \arg \min _{\mathbf{w}_{m}[l]} E\left[\left|\left\{\mathbf{w}_{m}[l]\right\}^{H} \mathbf{y}_{m}[l]\right|^{2}\right] \\
& \text { s.t. }\left\{\mathbf{w}_{m}[l]\right\}^{H} \mathbf{s}=1 \\
& l=N, \cdots, L-(N-1) .
\end{aligned}
$$


Using lagrangian multiplier method, the filter $\mathbf{w}_{m}[l]$ for the estimation of $\hat{\alpha}_{m}[l]$ can be derived as

$$
\mathbf{w}_{m}[l]=\frac{\left(E\left[\mathbf{y}_{m}[l] \mathbf{y}_{m}[l]^{H}\right]\right)^{-1} \mathbf{s}}{\mathbf{s}^{H}\left(E\left[\mathbf{y}_{m}[l] \mathbf{y}_{m}[l]^{H}\right]\right)^{-1} \mathbf{s}}
$$

We further assume the independence between scattering coefficients. As a consequence, the covariance matrix of observation $\mathbf{y}_{m}[l]$ can be written as

$$
E\left[\mathbf{y}_{m}[l] \mathbf{y}_{m}[l]^{H}\right]=\mathbf{G} \boldsymbol{\Pi}[l] \mathbf{G}^{H}+\sigma_{n}^{2} \mathbf{I}
$$

where $\sigma_{n}^{2}$ denotes the noise power and

$$
\boldsymbol{\Pi}[l]=\operatorname{diag}\left(\left[\left|\tilde{\alpha}_{m}[l-(N-1)]\right|^{2},, \ldots,\left|\tilde{\alpha}_{m}[l+(N-1)]\right|^{2}\right]\right)
$$

with $\tilde{\alpha}_{m}[l]$ the prior information of $\alpha_{m}[l]$, where we assume the target range profiles located different range cells are independent. Submitting Eq. (15) in Eq. (27), $\mathbf{w}_{m}[l]$ can be rewritten as

$$
\begin{aligned}
\mathbf{w}_{m}[l] & =\frac{\left(\mathbf{G} \Pi[l] \mathbf{G}^{H}+\sigma_{n}^{2} \mathbf{I}_{N}\right)^{-1} \mathbf{s}}{\mathbf{s}^{H}\left(\mathbf{G} \boldsymbol{\Pi}[l] \mathbf{G}^{H}+\sigma_{n}^{2} \mathbf{I}_{N}\right)^{-1} \mathbf{s}}, \\
l & =N, \cdots, L-(N-1)
\end{aligned}
$$

Applying $\mathbf{w}_{m}[l]$ to $\mathbf{y}_{m}[l]$, the estimation of $\alpha_{m}[l]$ is given by

$$
\hat{\alpha}_{m}[l]=\left(\mathbf{w}_{m}[l]\right)^{H} \mathbf{y}_{m}[l], \quad l=N, \cdots, L-(N-1) .
$$

As to the estimation of from 1 th to $(N-1)$ th range cells, i.e., $\hat{\alpha}_{m}[l], l=1, \cdots,(N-1)$, the observation of length $N$ is expressed as

$$
\mathbf{y}_{m}[N]=\mathbf{G a}_{m}[N]+\mathbf{b}_{m}[N]
$$

Similarly, the optimization problem can be given by,

$$
\begin{aligned}
& \arg \min _{\mathbf{w}_{m}[l]} E\left[\left|\left\{\mathbf{w}_{m}[l]\right\}^{H} \mathbf{y}_{m}[N]\right|^{2}\right] \\
& \text { s.t. }\left\{\mathbf{w}_{m}[l]\right\}^{H} \mathbf{g}_{l}=1 \\
& l=1, \cdots,(N-1),
\end{aligned}
$$

where $\mathbf{g}_{l}$ is the $l$ th column of $\mathbf{G}$. Consequently, the optimized filter $\mathbf{w}_{m}[l]$ of $l$ th range cell is derived as

$\mathbf{w}_{m}[l]=\frac{\left(\mathbf{G} \Pi[N] \mathbf{G}^{H}+\sigma_{n}^{2} \mathbf{I}_{N}\right)^{-1} \mathbf{g}_{l}}{\mathbf{g}_{l}^{H}\left(\mathbf{G} \Pi[N] \mathbf{G}^{H}+\sigma_{n}^{2} \mathbf{I}_{N}\right)^{-1} \mathbf{g}_{l}}, l=1, \ldots,(N-1)$.

We further obtain the estimation of $\alpha_{m}[l], l=1, \cdots$, $(N-1)$

$$
\hat{\alpha}_{m}[l]=\left(\mathbf{w}_{m}[l]\right)^{H} \mathbf{y}_{m}[N], l=1, \cdots,(N-1) .
$$

As to the estimation of from $(L-(N-2))$ th to $L$ th range cells, i.e., $\hat{\alpha}_{m}[l], l=L-(N-2), \cdots, L$, we write the observation $\mathbf{y}_{m}[L-(N-1)]$ as

$$
\mathbf{y}_{m}[L-(N-1)]=\mathbf{G a}_{m}[L-(N-1)]+\mathbf{b}_{m}[L-(N-1)]
$$

The optimization problem is

$$
\begin{aligned}
& \underset{\mathbf{w}_{m}[l]}{\arg \min } E\left[\left|\left\{\mathbf{w}_{m}[l]\right\}^{H} \mathbf{y}_{m}[L-(N-1)]\right|^{2}\right] \\
& \text { s.t. }\left\{\mathbf{w}_{m}[l]\right\}^{H} \mathbf{g}_{l-L+(2 N-1)}=1 \\
& l=L-(N-2), \cdots, L .
\end{aligned}
$$

Hence, the optimized filter coefficient $\mathbf{w}_{m}[l]$ is given by

$$
\begin{aligned}
\mathbf{w}_{m}[l] & =\frac{\left(\mathbf{G} \Pi[L-(N-1)] \mathbf{G}^{H}+\sigma_{n}^{2} \mathbf{I}_{N}\right)^{-1} \mathbf{g}_{l-L+(2 N-1)}}{\mathbf{g}_{l-L+(2 N-1)}^{H}\left(\mathbf{G} \Pi[L-(N-1)] \mathbf{G}^{H}+\sigma_{n}^{2} \mathbf{I}_{N}\right)^{-1} \mathbf{g}_{l-L+(2 N-1)}}, \\
l & =L-(N-2), \cdots, L .
\end{aligned}
$$

The estimation value of $\alpha_{m}[l], l=L-(N-2), \cdots, L$, can be computed as

$$
\hat{\alpha}_{m}[l]=\left(\mathbf{w}_{m}[l]\right)^{H} \mathbf{y}_{m}[L-(N-1)], l=L-(N-2), \cdots, L .
$$

Based on the above discussion, the estimation value $\left.\hat{\mathbf{a}}_{m}=\left[\hat{\alpha}_{m}[l], \ldots, \hat{\alpha}_{m}[L)\right]\right]^{T}, m=1, \cdots, M$ can be obtained. Hence, the estimation of $\hat{\mathbf{A}} \in \mathbb{C}^{L \times M}$ can be expressed as

$$
\hat{\mathbf{A}}=\left[\hat{\mathbf{a}}_{1}, \ldots, \hat{\mathbf{a}}_{M}\right] .
$$

\subsection{The process in Doppler dimension}

In this subsection, we focus on the process of doppler dimension in order to estimate the elements of rangeDoppler plane $\mathbf{X}$. Specifically, using the linear function in Eq. (8) and $\mathbf{A}[l]=\mathbf{X}[l] \mathbf{F}^{T}$, we have

$$
\hat{\mathbf{A}}=\mathbf{X} \mathbf{F}^{T}+\mathbf{E} \text {. }
$$

where $\mathbf{E}$ is noise vector with covariation matrix $\mathbf{I} / \mathbf{s}^{H} \mathbf{s}$. After transposition operation, Eq. (28) can be recast as,

$$
\hat{\mathbf{A}}^{T}=\mathbf{F} \mathbf{X}^{T}+\mathbf{E}^{T} \text {. }
$$

Let $\hat{\beta}[l], \mathbf{x}[l]$ and $\mathbf{e}[l]$ denote the $l$ th columns of $\hat{\mathbf{A}}^{T}, \mathbf{X}^{\mathrm{T}}$ and $\mathbf{E}^{T}$, respectively. Hence, similar to the process of range dimension, we have 


$$
\begin{aligned}
\hat{\beta}[l] & =\mathbf{F x}[l]+\mathbf{e}[l], \\
l & =1, \cdots, L,
\end{aligned}
$$

where $\hat{\beta}[l]$ and $\mathbf{x}[l]$ are $M \times 1$ and $K \times 1$ vectors, respectively.

Let $\mathbf{x}_{k}[l](k=1, \ldots, K)$ and $\mathbf{F}_{k}$ denote the $k$ th element of $\mathbf{x}[l]$ and $k$ th column of $\mathbf{F}$, respectively. The GCAPC filter coefficients $\mathbf{w}_{k}$ for the estimation of $\hat{\mathbf{x}}_{k}[l]$ are expressed as

$$
\mathbf{w}_{k}=\frac{\left(\mathbf{F D}[l] \mathbf{F}^{H}+\mathbf{I}_{M} / \mathbf{s}^{H} \mathbf{s}\right)^{-1} \mathbf{F}_{k}}{\mathbf{F}_{k}^{H}\left(\mathbf{F D}[l] \mathbf{F}^{H}+\mathbf{I}_{M} / \mathbf{s}^{H} \mathbf{s}\right)^{-1} \mathbf{F}_{k}},
$$

where

$$
\mathbf{D}[l]=\operatorname{diag}\left(\left[\left|\tilde{\mathbf{x}}_{1}[l]\right|^{2},, \ldots,\left|\tilde{\mathbf{x}}_{K}[l]\right|^{2}\right]\right) .
$$

$\tilde{x}_{k}[l]$ is the prior information of $x_{k}[l]$. Hence, the estimation value $\hat{\mathbf{x}}_{k}[l]$ can be obtained by

$$
\hat{\mathbf{x}}_{k}[l]=\left\{\mathbf{w}_{k}\right\}^{H} \hat{\beta}[l] .
$$

The estimation of $\hat{\mathbf{x}}[l]$ can be expressed as

$$
\hat{\mathbf{x}}[l]=\left[\hat{\mathbf{x}}_{1}[l], \ldots, \hat{\mathbf{x}}_{K}[l]\right]^{T} .
$$

Finally, we conduct the same procedure for each range cell and can achieve the estimation of $\hat{\mathbf{X}}$.

\subsection{The joint procedure of MAMPC algorithm}

In this subsection, the proposed procedure of MAMPC for the estimation of range-Doppler plane $\mathbf{X}$ is summarized in Algorithm 1.

Finally, it is worth highlighting that in each iteration, Algorithm 1 shares the computational complexity of $O\left(L M N^{3}+L K M^{3}\right)$. Additionally, we note that original AMPC [18] requires to conduct $N M \times N M$ matrix inversion for each individual range-Doppler cell with corresponding to $O\left((M N)^{3}\right)$ computation complexity. However, FAMPC [20] needs to implement $K_{l} K_{p} \times$ $K_{l} K_{p}$ sub-matrix inversion for each range-Doppler cell, which is order of $O\left(R_{p} R_{l}\left(K_{l} K_{p}\right)^{3}\right)$, where full-dimension model is divided into the $R_{l}$ segments in fast time domain and $R_{p}$ segments in slow time domain with $K_{l}=N / R_{l}$ and $K_{p}=M / R_{p}$. Compared with AMPC, the computation load of FAMPC algorithm reduces a factor of $\left(R_{p} R_{l}\right)^{2}$. In particular, MAMPC algorithm includes computational burden connected with $M$ matrix inversions with size of $N \times N$ and one matrix inversion with size of $M \times M$ for each range-Doppler cell, which is order of $O\left(M N^{3}+M^{3}\right)$. We note that MAMPC algorithm has the same order of computation load with FAMPC when $R_{p}=M, R_{l}=1$. Consequently, we conclude that FAMPC and MAMPC can significantly reduce the computational complexity in comparison with full-dimension AMPC algorithm.

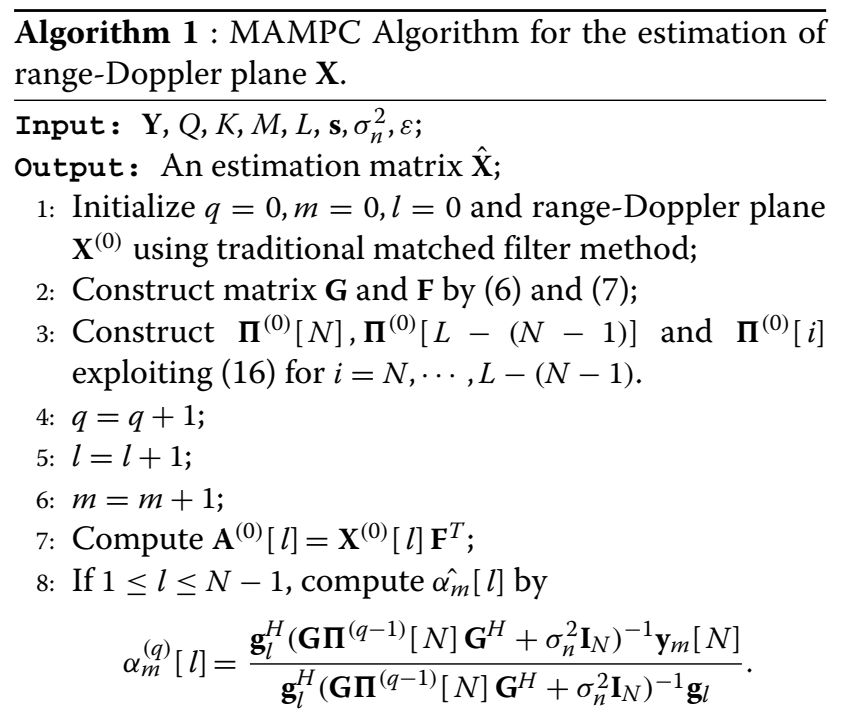

Otherwise, if $N \leq l \leq L-(N-1)$, compute $\hat{\alpha}_{m}[l]$ by

$$
\alpha_{m}^{(q)}[l]=\frac{\mathbf{s}^{H}\left(\mathbf{G} \boldsymbol{\Pi}^{(q-1)}[l] \mathbf{G}^{H}+\sigma_{n}^{2} \mathbf{I}_{N}\right)^{-1} \mathbf{y}_{m}[l]}{\mathbf{s}^{H}\left(\mathbf{G} \boldsymbol{\Pi}^{(q-1)}[l] \mathbf{G}^{H}+\sigma_{n}^{2} \mathbf{I}_{N}\right)^{-1} \mathbf{s}} ;
$$

or else, compute $\hat{\alpha}_{m}[l]$ by

$\alpha_{m}^{(q)}[l]=\frac{\mathbf{g}_{l-L+(2 N-1)}^{H}\left(\mathbf{G B}^{(q-1)} \mathbf{G}^{H}+\sigma_{n}^{2} \mathbf{I}_{N}\right)^{-1} \mathbf{y}_{m}[L-(N-1)]}{\mathbf{g}_{l-L+(2 N-1)}^{H}\left(\mathbf{G B}^{(q-1)} \mathbf{G}^{H}+\sigma_{n}^{2} \mathbf{I}_{N}\right)^{-1} \mathbf{g}_{l-L+(2 N-1)}}$,

where $\mathbf{B}^{(q-1)}=\Pi^{(q-1)}[L-(N-1)]$. The computational complexity involved in this step is in the order of $O\left(N^{3}\right)$.

9: If $m \geq M, m=0$ and return to step 10; otherwise, go to step 6;

10: If $l \geq L, l=0$ and go to step 11 ; otherwise go to step 5;

11: Construct $\mathbf{A}^{(q)}$ by using (27);

12: Initialize $n=0, k=0$;

13: $n=n+1$

14: $k=k+1$;

15: Construct $\mathbf{D}^{(q-1)}[n]$ using (32) and $\mathbf{X}^{(q-1)}$;

16: Construct $\beta^{(q)}[n]$ of $n$th column of $\mathbf{A}^{(q)^{T}}$;

17: Compute $\mathbf{x}_{k}^{(q)}[n]$ by

$$
\mathbf{x}_{k}^{(q)}[n]=\frac{\mathbf{F}_{k}^{H}\left(\mathbf{F D}^{(q-1)}[n] \mathbf{F}^{H}+\mathbf{I}_{M} / \mathbf{s}^{H} \mathbf{s}\right)^{-1} \beta^{(q)}[n]}{\mathbf{F}_{k}{ }^{H}\left(\mathbf{F D}^{(q-1)}[n] \mathbf{F}^{H}+\mathbf{I}_{M} / \mathbf{s}^{H} \mathbf{s}\right)^{-1} \mathbf{F}_{k}},
$$

which has computational complexity of $O\left(M^{3}\right)$.

18: If $k \geq K, k=0$ and return to step 19; otherwise, go to step 14;

19: If $n \geq L$, return to step 20; otherwise, go to step 13;

20: Construct $\mathbf{X}^{(q)}$ by employing (34);

21: Construct $\Pi^{(q)}[N], \Pi^{(q)}[L-(N-1)]$ and $\Pi^{(q)}[i]$ exploiting (16) for $i=N, \cdots, L-(N-1)$;

22: If $\frac{1}{L K}\left\|\mathbf{X}^{(q+1)}-\mathbf{X}^{(q)}\right\|^{2} \leq \varepsilon$, stop and output, where $\varepsilon$ is a user selected parameter to control convergence,; otherwise return to step 4. 


\section{The application of MAMPC algorithm in the presence of clutter}

In this section, we focus on the estimation of rangeDoppler plane $\mathbf{X}$ in the presence of clutter employing MAMPC algorithm. According to the received signal model in Eq. (8), we here adopt whitening method to remove correlation of clutter. Specifically, let $\mathbf{c}[l]=$ $\left[c_{1}[l], c_{2}[l], \cdots, c_{M}[l]\right]^{T}$ denote the range profile of the $l$ th cell for $M$ pulses; hence, we have

$$
\mathbf{C}[l]=\left[\begin{array}{c}
\mathbf{c}[l-(N-1)]^{T} \\
\mathbf{c}[l-(N-2)]^{T} \\
\vdots \\
\mathbf{c}[l+(N-1)]^{T}
\end{array}\right]
$$

In particular, we further assume that $c_{m}[j]$ and $c_{n}[i] \forall(m, n) \in\{1,2, \ldots, M\}^{2}, \forall(i, j) \in\{1,2, \cdots, L\}^{2}, i \neq$ $j$, are zero-mean uncorrelated random variables, and $c_{m}[j], c_{n}[i]$ with $i=j$ are correlative random variables obeying zero-mean Gaussian distribution with covariance matrix

$$
E\left[\mathbf{c}[l] \mathbf{c}^{H}[l]\right]=\sigma_{0} \mathbf{H}=\left(\sigma_{i, j}\right)_{M \times M}, \forall(i, j) \in\{1,2, \ldots, M\}^{2},
$$

where nonnegative number $\sigma_{0}$ is the power of clutter, $\mathbf{H}$ is the positive semidefinite Hermitian-Toeplitz matrix with size $M \times M$, whose main diagonal elements 1 .

Our purpose is to remove correlation of clutter. In other words, the interference term including clutter and noise in Eq. (8) should show the same statistics feature as the noise term in Eq. (8) after whitening operation. In particular, we stack all the column of $\mathbf{Y}[l]$ with only considering the interference term in Eq. (8), denoted as $\tilde{\mathbf{Y}}[l]$, i.e., $\tilde{\mathbf{Y}}[l]=$ $\left[\mathbf{y}_{1}^{T}[l], \ldots, \mathbf{y}_{M}^{T}[l]\right]^{T}$. Hence, we have

$$
\begin{aligned}
& E\left[\tilde{\mathbf{Y}}[l] \tilde{\mathbf{Y}}^{H}[l]\right]= \\
& {\left[\begin{array}{cccc}
\mathbf{G} E\left[\mathbf{c}_{1}[l] \mathbf{c}_{1}[l]^{H}\right] \mathbf{G}^{H} & \mathbf{G} E\left[\mathbf{c}_{1}[l] \mathbf{c}_{2}[l]^{H}\right] \mathbf{G}^{H} & \ldots & \mathbf{G} E\left[\mathbf{c}_{1}[l] \mathbf{c}_{M}[l]^{H}\right] \mathbf{G}^{H} \\
\mathbf{G} E\left[\mathbf{c}_{2}[l] \mathbf{c}_{1}[l]^{H}\right] \mathbf{G}^{H} & \mathbf{G} E\left[\mathbf{c}_{2}[l] \mathbf{c}_{2}[l]^{H}\right] \mathbf{G}^{H} & \ldots & \mathbf{G} E\left[\mathbf{c}_{2}[l] \mathbf{c}_{M}[l]^{H}\right] \mathbf{G}^{H} \\
\vdots & \vdots & \ddots & \vdots \\
\mathbf{G} E\left[\mathbf{c}_{M}[l] \mathbf{c}_{1}[l]^{H}\right] \mathbf{G}^{H} & \mathbf{G} E\left[\mathbf{c}_{M}[l] \mathbf{c}_{2}[l]^{H}\right] \mathbf{G}^{H} & \ldots & \mathbf{G} E\left[\mathbf{c}_{M}[l] \mathbf{c}_{M}[l]^{H}\right] \mathbf{G}^{H}
\end{array}\right]} \\
& +\sigma_{n}^{2} \mathbf{I}_{N M}
\end{aligned}
$$

Exploiting the fact in Eq. (36) that $\mathbf{G E}\left[\mathbf{c}_{i}[l] \mathbf{c}_{j}[l]^{H}\right]$ $\mathbf{G}^{H}=\sigma_{i, j} \mathbf{G G}^{H}, i, j \in\{1,2, \ldots, M\}^{2}$, the whitening matrix $E\left[\tilde{\mathbf{Y}}[l] \tilde{\mathbf{Y}}^{H}[l]\right]^{-\frac{1}{2}}[22]$ can be given by,

$$
\begin{aligned}
& E\left[\tilde{\mathbf{Y}}[l] \tilde{\mathbf{Y}}^{H}[l]\right]^{-\frac{1}{2}} \\
& =\left[\sigma_{0}(\mathbf{H} \otimes \mathbf{R})+\sigma_{n}^{2} \mathbf{I}_{N M}\right]^{-\frac{1}{2}}
\end{aligned}
$$

where $\mathbf{R}=\mathbf{G G}^{H}$ is the correlation matrix for the range dimension, computed as

$$
\mathbf{R}=\left[\begin{array}{cccc}
r_{s}[0] & r_{s}[-1] & \cdots & r_{s}[-(N-1)] \\
r_{s}[1] & r_{s}[0] & \cdots & r_{s}[-(N-2)] \\
\vdots & \vdots & \ddots & \vdots \\
r_{s}[N-1] & r_{s}[N-2] & \cdots & r_{s}[0]
\end{array}\right]
$$

with $r_{s}[n], n=-N+1, \cdots, N+1$ being the autocorrelation value of $\mathrm{s}$ at delay $n$.

Assuming that $\mathrm{s}$ possesses good autocorrelation property, i.e., $r_{s}[n] \ll r_{s}[0], n=-(N-1), \ldots, N-1, n \neq 0$, we have

$$
\mathbf{R} \approx r_{s}[0] \mathbf{I}_{N}
$$

Submitting Eq. (40) into Eq. (38), we have

$$
\begin{aligned}
& E\left[\tilde{\mathbf{Y}}[l] \tilde{\mathbf{Y}}^{H}[l]\right]^{-\frac{1}{2}} \\
& \approx\left[\sigma_{0}\left(\mathbf{H} \otimes \mathbf{I}_{N}\right)+\sigma_{n}^{2} \mathbf{I}_{N M}\right]^{-\frac{1}{2}}
\end{aligned}
$$

where we suppose that the energy of s equals to 1 .

Based on the aforementioned discussion, the whitening result $\tilde{\mathbf{Y}}_{W}[l]$ of $\tilde{\mathbf{Y}}[l]$ can be expressed as follows

$$
\tilde{\mathbf{Y}}_{W}[l]=\left(\sigma_{0}\left(\mathbf{H} \otimes \mathbf{I}_{N}\right)+\sigma_{n}^{2} \mathbf{I}_{N M}\right)^{-1 / 2} \tilde{\mathbf{Y}}[l] .
$$

According to Eq. (42), we can obtain the whitening result of $\mathbf{Y}[l]$ through some mathematical operations, given by

$$
\mathbf{Y}_{W}[l]=\mathbf{Y}[l]\left(\sigma_{0} \mathbf{H}^{T}+\sigma_{n}^{2} \mathbf{I}_{M}\right)^{-1 / 2} .
$$

It is worth noting that decorrelation of observation matrix can be achieved by right-multiplying the whitening matrix $\boldsymbol{\Gamma}=\left(\sigma_{0} \mathbf{H}^{T}+\sigma_{n}^{2} \mathbf{I}_{M}\right)^{-1 / 2}$, which decreases the computation load.

Finally, exploiting Eq. (43), Eq. (8) can be expressed as

$$
\mathbf{Y}_{1}[l]=\mathbf{G X}[l] \mathbf{F}_{1}^{T}+\mathbf{B}_{1}[l]
$$

where $\mathbf{Y}[l] \mathbf{\Gamma}=\mathbf{Y}_{1}[l], \mathbf{F}^{T} \boldsymbol{\Gamma}=\mathbf{F}_{1}^{T}, \mathbf{G C}[l] \mathbf{\Gamma}+\mathbf{B}[l] \mathbf{\Gamma}=$ $\mathbf{B}_{1}[l]$ with the same statistical characteristics as $\mathbf{B}[l]$.

Finally, we can estimate $\mathbf{X}[l]$ based on $\mathbf{Y}_{1}[l]$ using MAMPC Algorithm which is reported in 3.

\section{Numerical results}

In this section, we assess the performance of proposed algorithm for the estimation of range-Doppler plane $\mathbf{X}$ in terms of clutter-free scenario and clutter scenario. To this end, we consider a multi-targets case with corresponding to locations, velocities, and signal-to-noise radio (SNR) of targets given in Table 1 . Besides, we suppose the range processing window $L=100$ and the number of Doppler cell $K=128$ and consider the transmit signal ${ }^{1}$ is linear frequency modulation (LFM) phase coding with code 
Table 1 Target parameters

\begin{tabular}{llll}
\hline Range cell index & Velocity $(\mathrm{m} / \mathrm{s})$ & $\begin{array}{l}\text { Normalized Doppler } \\
\text { frequency }\end{array}$ & SNR (dB) \\
\hline 40 & 30 & 0.2 & 5 \\
30 & 35 & 0.233 & -5 \\
45 & -35 & -0.233 & 10 \\
47 & -40 & -0.267 & 0 \\
60 & 35 & 0.233 & -8 \\
55 & 30 & 0.2 & 5 \\
70 & -30 & -0.2 & 5 \\
25 & -30 & -0.2 & -5 \\
20 & 22 & 0.147 & -8 \\
30 & 62 & 0.413 & -5 \\
\hline
\end{tabular}

length $N=32$, bandwidth $B=4 \mathrm{MHz}$, pulse width $T=$ $4 \mu \mathrm{s}$, center frequency $f_{0}=\mathrm{GHz}$, and PRT $T_{P R T}=1 \mathrm{~ms}$. In partiuclar, we set the pulse number $M=32$. Finally, we consider the exit condition $\varepsilon=10^{-6}$ for Algorithm 1 . Besides, the running computation time is analyzed using Matlab 2010a version, running on a standard PC (with a $3.3 \mathrm{GHz}$ Core i5 CPU and 8 GB RAM).

\subsection{Clutter-free scenario}

In this subsection, we focus on the discussion of MAMPC in terms of achieved estimation of range-Doppler plane $\mathbf{X}$ and computational burden accounting for clutter-free scenario. In particular, for comparison purpose, matched filter and MTD (MF-MTD), AMPC, and FAMPC are also evaluated.

Figure 1 exhibits the estimation of range-Doppler plane $\mathbf{X}$ using traditional MF-MTD. In particular, the

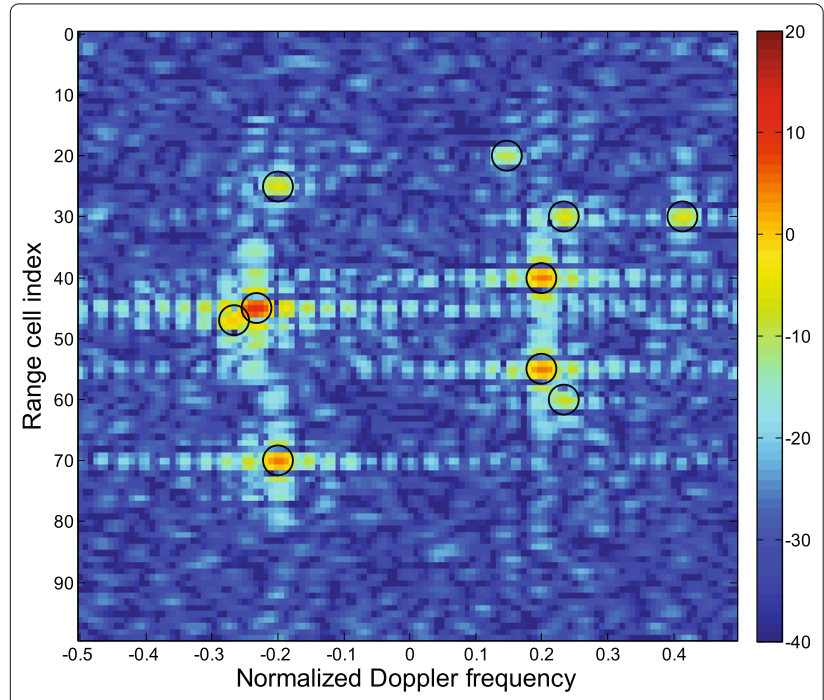

Fig. 1 The estimation (in dB) of range-Doppler plane X using traditional matched filter and MTD method for clutter-free scenario locations of true targets are marked with circles. The results indicate that weak targets are possibly masked by the range-Doppler sidelobes of strong targets. For example, the high sidelobe of target at the 45th range cell has a significant impact on parameter estimating (i.e., range profile) of nearby target located at the 47th range cell. It could be treated as a weak target leading to false alarm. Additionally, the mainlobes of the targets are expanded over the range-Doppler plane.

Based on the APC and GCAPC, Figs. 2, 3, and 4 depict the estimations of range-Doppler plane $\mathbf{X}$ utilizing AMPC, FAMPC, and MAMPC, respectively. In particular, we observe that, in Figs. 2a, 3a, and 4a, the targets can be accurately estimated by exploiting AMPC, FAMPC, and MAMPC based on the accomplishment of GCAPC. However, the obtained results using FAMPC also exhibit the mainlobe energy of the targets can easily spread nearby-range Doppler cells, as well as possess high-range Doppler sidelobes, low range, and Doppler resolutions in comparison with those optimized by AMPC and MAMPC. Additionally, in Figs. 2b, 3b, and $4 \mathrm{~b}$, we assess the obtained estimation of range-Doppler plane $\mathbf{X}$ exploiting AMPC, FAMPC, and MAMPC accomplished by APC. Interestingly, AMPC, FAMPC, and MAMPC achieve lower range-Doppler sidelobe and narrower mainlobe in terms of estimation results of range-Doppler plane $\mathbf{X}$ compared with MFMTD, whereas a portion of weak targets are missing for FAMPC.

In the following, we analyze the mean square error (MSE) performance of the estimation using MF-MTD, AMPC, FAMPC, and MAMPC. In particular, the MSE is defined as

$$
M S E=\frac{1}{L K}\|\hat{\mathbf{X}}-\mathbf{X}\|^{2}
$$

In Fig. 5, we plot the MSE curves of the $\mathbf{X}$ estimation versus iteration number exploiting MF-MTD, AMPC, FAMPC, and MAMPC based on GCAPC for clutter-free scenario. Interestingly, AMPC and MAMPC both share the near performance and outperform MF-MTD and FAMPC. This is a reasonable behavior since the optimized results by MF-MTD and FAMPC show high range-Doppler sidelobes (Figs. 1 and 3a).

In Table 2, we report the iteration number and computation time of AMPC, FAMPC, and MAMPC for the implementation of estimation of range-Doppler plane $\mathbf{X}$ in clutter-free scenario. As expected, MAMPC outperforms AMPC and FAMPC in terms of computation time. Specifically, MAMPC costs $7.7 \mathrm{~s}$ to the estimation, whereas AMPC and FAMPC require 1014.7 and $91.6 \mathrm{~s}$, 

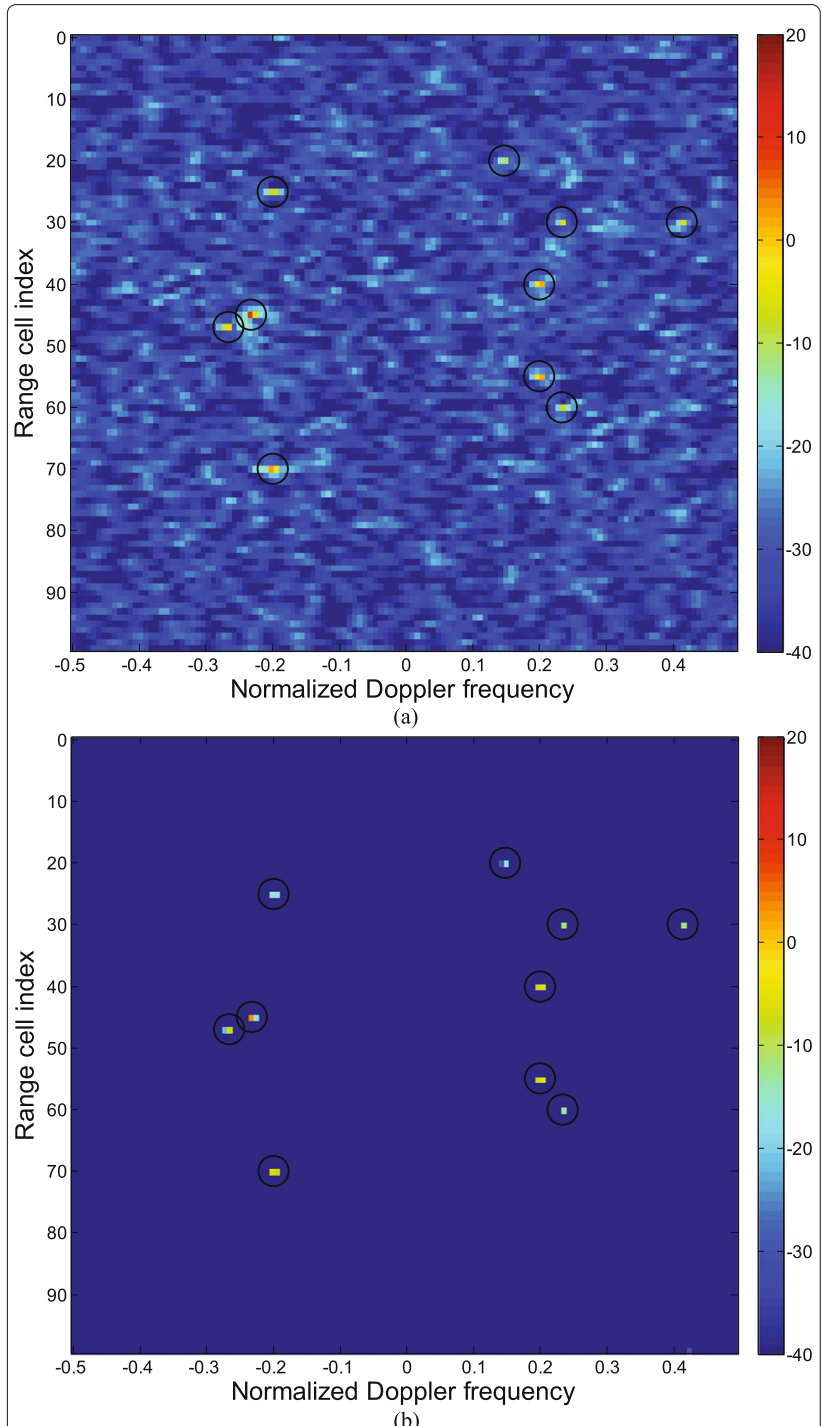

(b)

Fig. 2 The estimation (in dB) of range-Doppler plane X using AMPC for clutter-free scenario. a GCAPC. b APC

respectively. Finally, it is worth highlighting that MAMPC achieves the significant reduce of computational burden in comparison with AMPC and obtains more accurate estimation of range-Doppler plane $\mathbf{X}$ in contrast to FAMPC.

\subsection{Clutter scenario}

In this subsection, we consider the estimation of rangeDoppler plane $\mathbf{X}$ in presence of clutter, where we suppose that colored Gaussian clutter is adopted with assuming internal motion of the clutter scatters due to, for example, wind affecting a forest or grassland. Thus, the temporal correlation of such clutter can be described by its power spectral density (PSD),

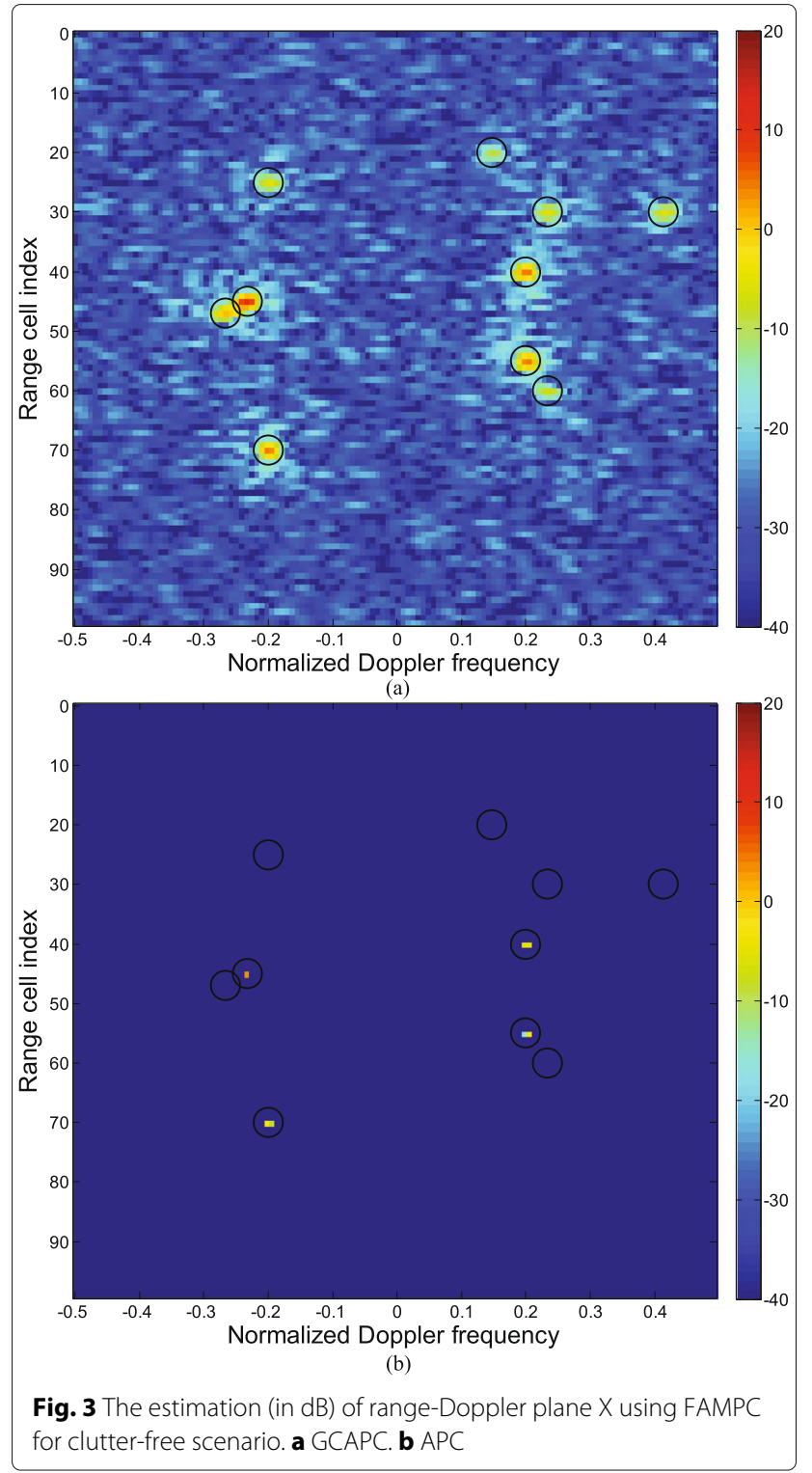

$$
S_{c}(f)=\frac{\sigma_{c}^{2} \lambda}{\sqrt{2 \pi} 2 \sigma_{v}} \exp \left(\frac{-f^{2} \lambda^{2}}{8 \sigma_{v}^{2}}\right),
$$

where $\sigma_{v}$ is the root of mean square(rms) of clutter velocity and $\lambda$ is the length of waveform. Furthermore, the autocorrelation function of clutter is expressed as

$$
r_{c}(\tau)=\sigma_{c}^{2} \exp \left(\frac{-\pi^{2} \tau^{2} 8 \sigma_{v}^{2}}{\lambda^{2}}\right) .
$$

Hence, the $(i, j)$ th element of the covariance matrix $\sigma_{0} \mathbf{H}$ is $\sigma_{i, j}=r_{c}\left(|i-j| T_{P R T}\right) / \sigma_{c}^{2}$ for $i, j=1, \ldots, M$. Here, we assume the rms of clutter $\sigma_{v}=5 \mathrm{~m} / \mathrm{s}$ and the power of clutter $P=\sigma_{c}^{2}=30 \mathrm{~dB}, \lambda=c / f_{0}=0.3 \mathrm{~m}$ with $c=$ $3 \times 10^{8} \mathrm{~m} / \mathrm{s}$ being the velocity of light. 


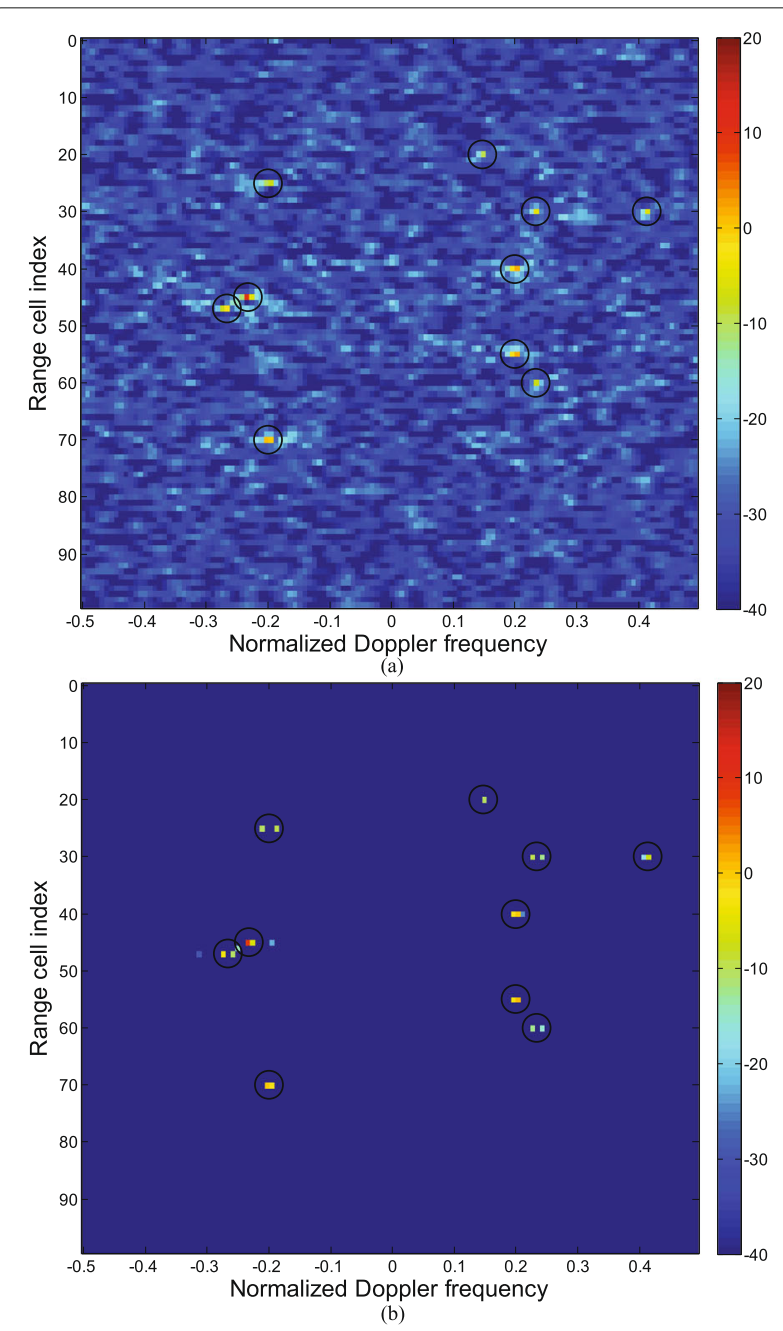

Fig. 4 The estimation (in $\mathrm{dB}$ ) of range-Doppler plane $X$ using MAMPC for clutter-free scenario. a GCAPC. b APC

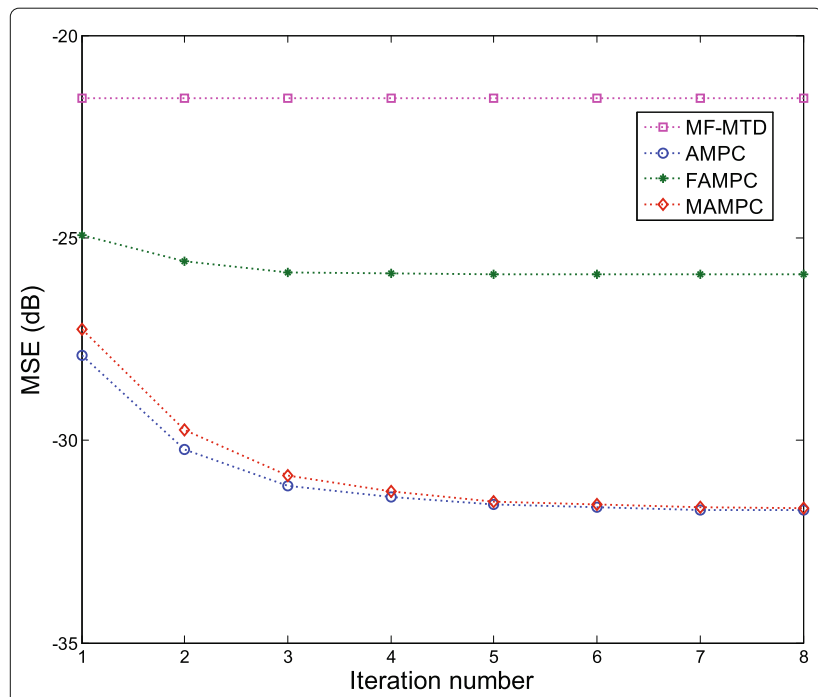

Fig. 5 The MSE (dB) versus iteration number using MF-MTD, AMPC, FAMPC, and MAMPC based on GCAPC for clutter-free scenario
Table 2 Iteration number and computation time (in seconds) of AMPC, FAMPC, and MAMPC for clutter-free scenario

\begin{tabular}{llll}
\hline Algorithm & AMPC & FAMPC & MAMPC \\
\hline$n$ & 6 & 4 & 7 \\
Time(s) & 1014.7 & 91.6 & 7.7 \\
\hline
\end{tabular}

In Fig. 6, the obtained range-Doppler plane $\mathbf{X}$ by traditional MF-MTD for clutter scenario is plotted. In particular, we observe that the targets completely are masked by strong clutter, showing that the adopted method fails to the considered clutter scenario.

In Figs.7a, 8a, and 9a, we plot the estimation results of range-Doppler plane $\mathbf{X}$ obtained by AMPC, FAMPC, and MAMPC utilizing GCAPC, respectively. Interestingly, it can be seen that the weak target located at 20th range cell, cannot be found by AMPC, FAMPC, and MAMPC. Again, a wide mainlobe behavior can be observed for FAMPC. Based on the accomplishment of APC, we give the estimation results of range-Doppler plane $\mathbf{X}$ obtained by AMPC, FAMPC, and MAMPC in Figs. 7b, 8b, and 9b. Results again show that FAMPC is noneffective to a part of weak targets. In particular, by contrasting to MF-MTD, AMPC, and MAMPC can attain to a much better estimation and significantly alleviate the impact of clutter.

In Fig. 10, the MSE curves of the $\mathbf{X}$ estimation versus iteration number exploiting MF-MTD, AMPC, FAMPC, and MAMPC based on GCAPC for clutter scenario, are plotted. As expected, MAMPC exhibits a slightly

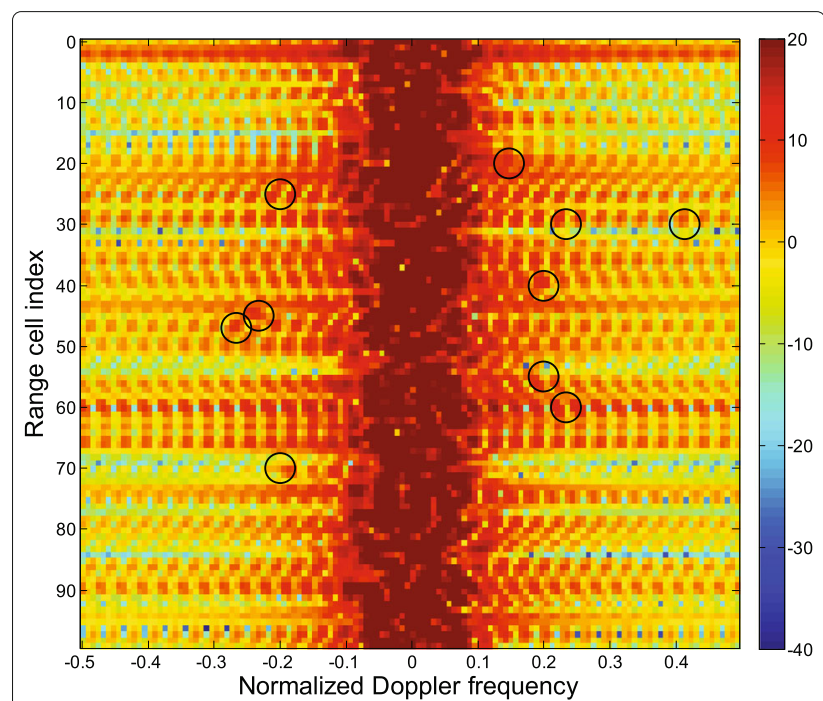

Fig. 6 The estimation (in dB) of range-Doppler plane X using traditional matched filter and MTD method for clutter scenario 

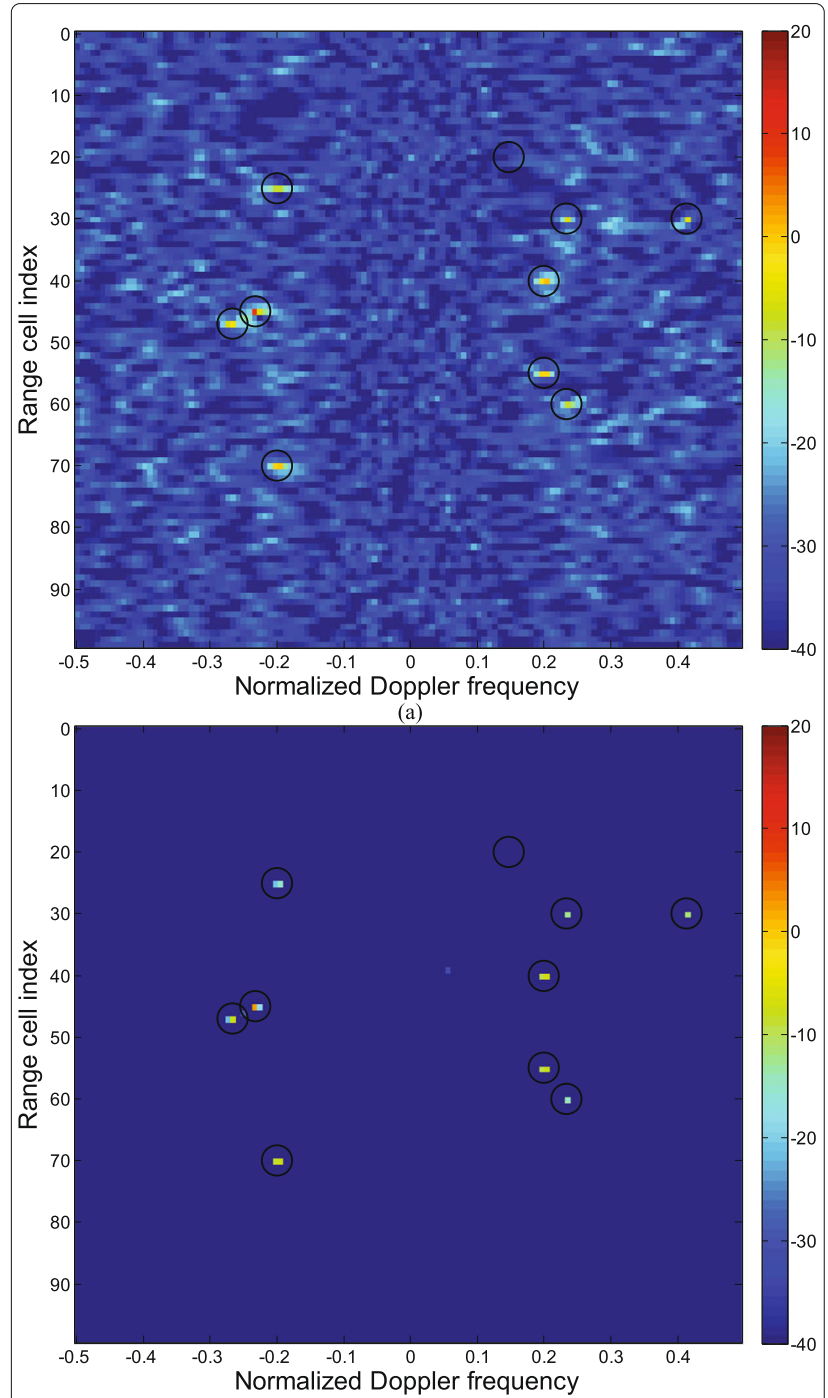

(b)

Fig. 7 The estimation (in $\mathrm{dB}$ ) of range-Doppler plane X using AMPC for clutter scenario. a GCAPC. b APC
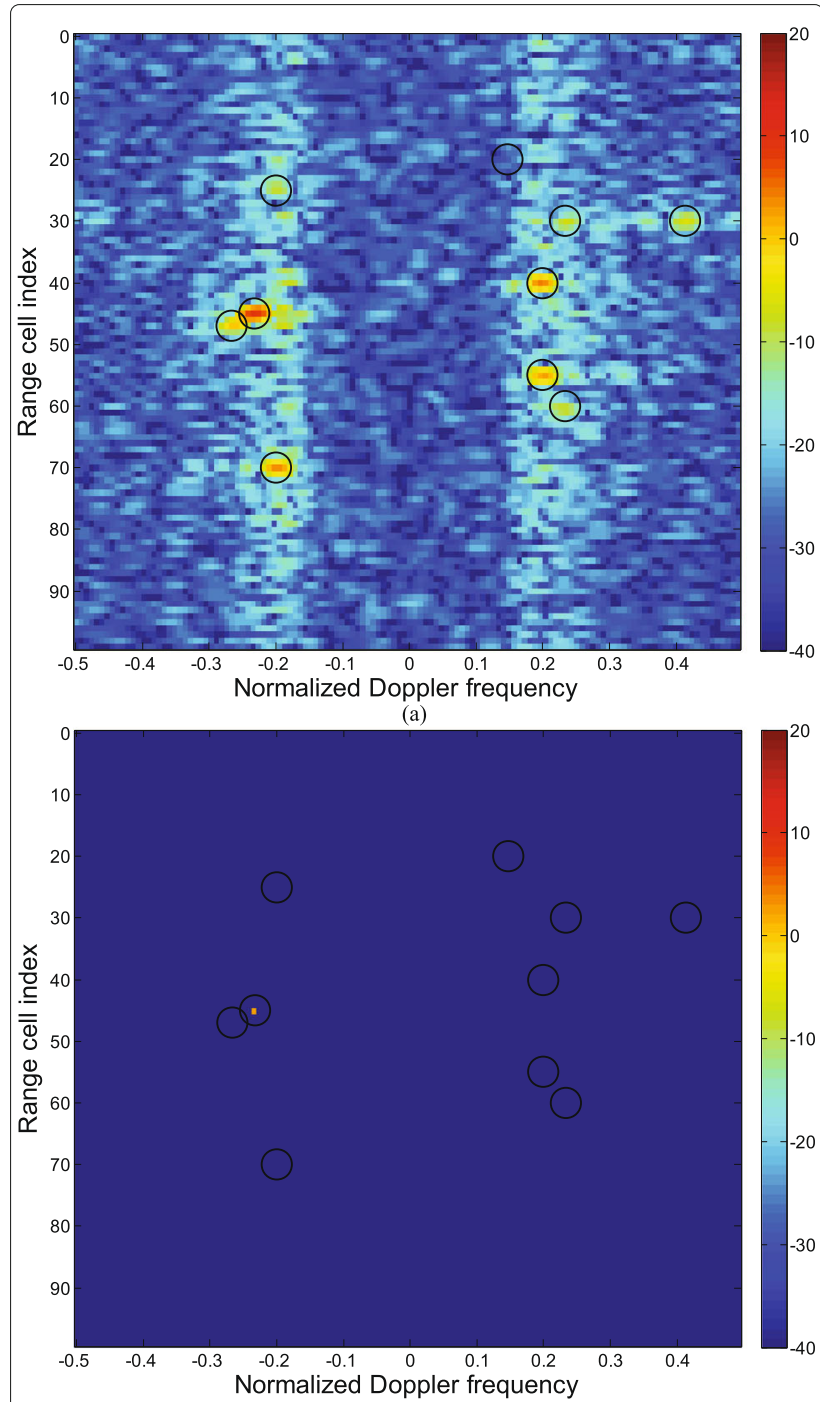

(b)

Fig. 8 The estimation (in dB) of range-Doppler plane X using FAMPC for clutter scenario. a GCAPC. b APC better performance than AMPC, and outperform MFMTD and FAMPC due to low range-Doppler sidelobes in Fig. 9(a).

Table 3 summarizes the behavior of computational time of AMPC, FAMPC, and MAMPC for clutter scenario. Again, MAMPC exhibits a lower computation burden than AMPC and FAMPC. Precisely, MAMPC spends 7.1s to implement the estimation, whereas AMPC and FAMPC need 1400.5 and 105.8 s, respectively. Finally, it is worth pointing out that the performance behaviors in Fig. 10 and in Table 2 reflect the capability of the proposed MAMPC that not only estimates range-Doppler plane $\mathbf{X}$ accurately, but also can reduce significantly computation load.

\section{Conclusions}

In this paper, we have addressed the estimation of range-Doppler plane for pulse Doppler radar systems considering clutter-free scenario and clutter scenario. We have proposed MAMPC algorithm including the estimation stages of range dimension and Doppler dimension for clutter-free scenario, where each stage is implemented based on GCAPC. In addition, we also have presented the combination of whitening method removing the correlation of the clutter component and MAMPC algorithm for considered clutter scenario. We have designed numerical simulations to assess the ability of proposed algorithm. We have observed that the proposed MAMPC keeps the near same estimation performance of range-Doppler 

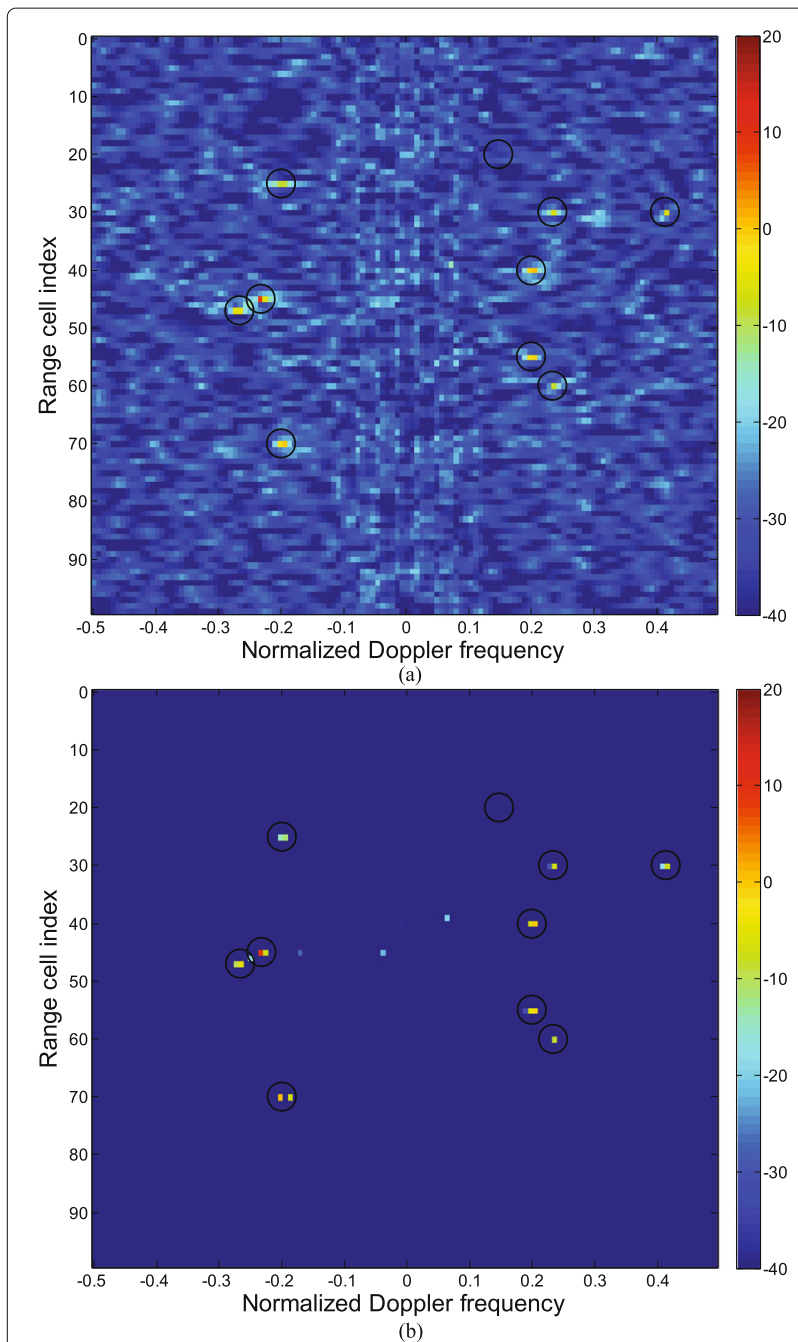

Fig. 9 The estimation (in dB) of range-Doppler plane X using MAMPC for clutter scenario. a GCAPC. b APC

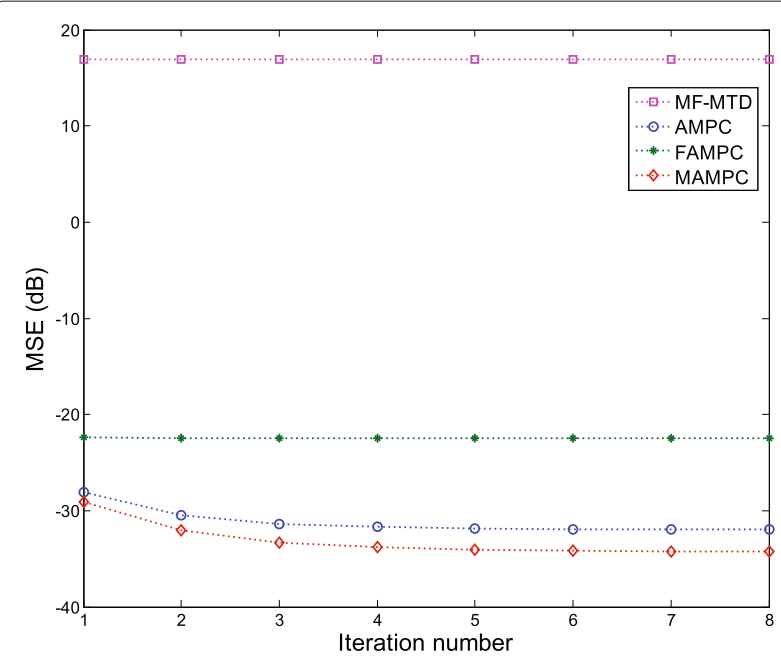

Fig. 10 The MSE (dB) versus iteration number using MF-MTD, AMPC, FAMPC, and MAMPC based on GCAPC for clutter scenario
Table 3 Iteration number and computation time (in seconds) of AMPC, FAMPC, and MAMPC for clutter scenario

\begin{tabular}{llll}
\hline Algorithm & AMPC & FAMPC & MAMPC \\
\hline$n$ & 8 & 4 & 6 \\
Time(s) & 1400.5 & 105.8 & 7.1 \\
\hline
\end{tabular}

plane $\mathbf{X}$ with that of AMPC, whereas FAMPC is likely to lose weak targets. Results have also impled that MAMPC shares much less computational time in comparision with AMPC. Possible future research tracks might concern the extension of the proposed framework to account for electronic jamming and nonhomogeneous characteristics clutter.

\section{Endnote}

${ }^{1}$ We notice that the waveform with good autocorrelation has better estimation performance for range-Doppler plane. Since the limitation on paper length, we here do not show the simulation results for the selection of waveform in simulation.

\section{Acknowledgements}

This work was supported by the National Natural Science Foundation of China under Grants 61201276 and 61301266, the Fundamental Research Funds of Central Universities under Grants ZYGX2014J013 and ZYGX2014Z005.

\section{Competing interests}

The authors declare that they have no competing interests.

\section{Author details}

${ }^{1}$ University of Electronic Science and Technology of China, 2006 Xi Yuan Ave, West Hi-tech Zone, Chengdu, China. ${ }^{2} \mathrm{AVIC}$ Leihua Electronic Technology Research Institute, Wuxi, China. ${ }^{3} 7306$ Research Institute of CASC, Chengdu, China.

Received: 25 June 2016 Accepted: 9 November 2016 Published online: 26 November 2016

\section{References}

1. MI Skolnik, Introduction to Radar Systems, 3rd edn. (McGraw-Hill, New York, 2001), pp. 1010-1014

2. S Nagand, M Barnes, in Proceedings of the 2003 IEEE Radar Conference. A moving target detection filter for an ultrawideband radar (Radar Conference, Huntsville, 2003), pp. 147-153

3. B Zrnic, A Zejak, A Petrovic, I Simic, Range sidelobe suppression for pulse compression radars utilizing modified RLS algorithm. 5th IEEE Int. Symp. Spread Spectrum Tech. Appl. 3, 1008-1011 (1998)

4. R Sato, M Shinrhu, Simple mismatched filter for binary pulse compression code with small PSL and small S/N loss. IEEE Trans. Aerosp. Electron. Syst. 39(2), 711-718 (2003)

5. SD Blunt, K Gerlach, Adaptive pulse compression via MMSE estimation. IEEE Trans. Aerosp. Electron. Syst. 42(2), 572-584 (2006)

6. L Li, W Yi, L Kong, XB Yang, in Proceedings of 2014. Range limited adaptive pulse compression via linear bayes estimation (IEEE Radar Conference, Cincinnati, 2014), pp. 1010-1014

7. THiggins, SD Blunt, K Gerlach, in Proceedings of 2009. Gain-constrained adaptive pulse compression via an MVDR framework (IEEE Radar Conference, Pasadena, 2009), pp. 1-6

8. RO Schmidt, Multiple emitter location and signal parameter estimation IEEE Trans. Ant. Pro. 34(3), 276-280 (1986)

9. A Barabell, in Acoustics, Speech, and Signal Processing, IEEE International Conference on ICASSP'83. Improving the resolution performance of 
eigenstructure-based direction-finding algorithms, vol. 8 (ICASSP, Boston, 1983), pp. 336-339

10. A Paulraj, R Roy, T Kailath, A subspace rotation approach to signal parameter estimation. Proc. IEEE. 74(7), 1044-1046 (1986)

11. SD Blunt, $T$ Chan, $K G$, in Proceedings of the IEEE Sensor Array and Multichannel Signal Processing Workshop. SAM 2008. 5th IEEE. A new framework for direction of-arrival estimation (SAM, Darmstadt, 2008), pp. $81-85$

12. SD Blunt, $T$ Chan, K Gerlach, Robust doa estimation: the reiterative superresolution (RISR) algorithm. IEEE Trans. Aerosp. Electron. Syst. 47(1), 332-346 (2011)

13. JM Baden, MN Cohen, in Proceedings of Record of the IEEE 1990 International Radar Conference. Optimal peak sidelobe filters for biphase pulse compression (IEEE Radar Conference, 1990), pp. 249-252

14. JM Baden, MN Cohen, in Proceedings of the National Telesystems Conference. Optimal sidelobe suppression for biphase codes, vol. (NTC, Georgia World Congress Center, Atlanta, 1991), pp. 127-131

15. S Wang, Z Li, Y Zhang, Application of optimized filters to two-dimensional sidelobe mitigation in meteorological radar sensing. IEEE Geosci. Remote Sens. Lett. 9(4), 778-782 (2012)

16. RC Chen, THiggins, in Proceedings of 2010 International Waveform Diversity and Design Conference (WDD). Golay waveforms and adaptive estimation, (WDD, Niagara Falls, 2010), pp. 257-261

17. THiggins, SD Blunt, AK Shackelford, in Proceedings of 2010 International Waveform Diversity and Design Conference (WDD). Time-range adaptive processing for pulse agile radar, (WDD, Niagara Falls, 2010), pp. 115-120

18. B Zhao, LJ Kong, M Yang, GL Cui, in Proceedings of 2011 IEEE CIE International Conference on Radar. Range-Doppler sidelobe and clutter suppression via time range adaptive processing (IEEE Radar (Radar), Chengdu, 2011)

19. SD Blunt, T Higgins, in Proceedings of 2007 IEEE Radar Conference. Achieving real-time efficiency for adaptive radar pulse compression (IEEE Radar Conference, Boston, 2007), pp. 116-121

20. L Kong, M Yang, B Zhao, in Proceedings of 2012 IEEE Radar Conference. Fast implementation of adaptive multi-pulse compression via dimensionality reduction technique (IEEE Radar Conference (RADAR), Atlanta, 2012), pp. 0435-0440

21. Y Yang, L Li, G Cui, W Yi, L Kong, X Yang, in 2015 IEEE Radar Conference. A modified adaptive multi-pulse compression algorithm for fast implementation (IEEE Radar Conference (RadarCon), Arlington, 2015), pp. 0390-0394

22. SM Kay, Fundamentals of Statistical Precessing, 2nd edn. (Publishing House of Electronics Industry, Bei Jing, 2011)

\section{Submit your manuscript to a SpringerOpen ${ }^{\circ}$ journal and benefit from:}

- Convenient online submission

- Rigorous peer review

- Immediate publication on acceptance

- Open access: articles freely available online

- High visibility within the field

- Retaining the copyright to your article

Submit your next manuscript at $\gg$ springeropen.com 